\title{
Code-Division Multiple Transmission for High-Speed UWB Radar Imaging With an Antenna Array
}

\author{
Takuya Sakamoto, Member, IEEE, and Toru Sato, Member, IEEE
}

\begin{abstract}
The ultrawideband (UWB) radar is a promising high-resolution 3-D imaging technique for near targets. We have developed a high-speed imaging algorithm, SEABED, for a UWB pulse radar, a key real-time imaging technology. When the algorithm is applied to UWB, antenna scanning for data acquisition takes significantly longer than calculating the SEABED algorithm itself. This presents a serious problem for the real-time application of UWB radar. In this paper, we use pseudonoise (PN) sequences as the transmitting waveforms, while the original work on the SEABED algorithm assumed impulsive short-wave pulses. Using PN sequences enables us to simultaneously transmit signals with multiple antennas, eliminating the need to scan antennas. We demonstrate that the proposed radar system works well using random sequences to suppress direct waves, which is critical in achieving high speeds for imaging.
\end{abstract}

Index Terms-Code division, gold sequence, imaging, SEABED algorithm, ultrawideband (UWB) radar.

\section{INTRODUCTION}

$\mathbf{S}$ INCE the Federal Communications Commission (FCC) standardized ultrawideband (UWB) for commercial use in the U.S. in 2002, the UWB radar has been a promising highresolution imaging tool for indoor and outdoor environments. A UWB radar can be applied in a variety of applications including imaging for robotics and security systems. Imaging with UWB radar shares many characteristics with conventional ground penetrating radar (GPR) technologies, whose algorithms can also be applied to UWB radar imaging [1]-[6]. However, it is difficult to directly apply techniques developed for conventional GPR techniques to produce real-time imaging because recursive and repetitive procedures are generally required. To resolve this problem, a simplified model of the target shape can prove useful. Because most targets in the air have clear boundaries, conventional models derived from GPR and nonreal-time imaging are unnecessarily complicated with too many degrees of freedom. Previous work developed a high-speed radar imaging algorithm, SEABED, with a simplified target model [7]-[16]. The SEABED algorithm estimates the target image using a reversible transform between the target shape and the observed data, assuming that all targets have clear

Manuscript received July 17, 2008; revised October 20, 2008. First published March 6, 2009; current version published March 27, 2009.

The authors are with the Department of Communications and Computer Engineering, Graduate School of Informatics, Kyoto University, Kyoto 606-8501, Japan.

Digital Object Identifier 10.1109/TGRS.2008.2009314 boundaries. It has been applied in through-the-wall imaging [17] and breast cancer detection [18], [19].

The SEABED algorithm can estimate target shapes quickly. The data acquisition time, however, has not previously been considered in evaluating the speed of systems employing the SEABED algorithm. High-speed imaging requires both fast measurement and signal processing. Many conventional studies have, however, used time-consuming antenna scanning in systems intended for high-speed performance [20], [21]. One solution to problems of slow scanning speed is to use a receiver array with a single transmitter. The use of receiver arrays in UWB GPR imaging has been well studied [22]. Another approach is the use of multiple transmitters with a single receiver. Both of these techniques can be used at the same time to increase the effective number of sensors as per [23] and [24] and suit the use of the SEABED algorithm [25]. The multiple transmission scheme in [25] requires sequential transmissions with multiple RF switches to select a transmitting antenna from the array of antennas. This is because a short pulse is used as a transmitted signal. In seeking to speed up data acquisition, we cannot transmit multiple short pulse signals simultaneously with the transmitting antennas, as they will be indistinguishable from each other. In this paper, we overcome this problem by using pseudonoise (PN) signals and multiple transmitters, making it possible to send multiple distinct transmissions with a switchfree simple radar system. This high-speed option compliments the speed of the SEABED algorithm, while conventional RF switching, as used in [25], is slow, easily neglecting the speed gains that the algorithm provides. Ishigami et al. [26] proposed a code-division simultaneous transmission system for ultrasonic imaging, which has been applied to medical use. Their method uses Walsh codes orthogonal to each other where echoes are completely synchronized. This is not realistic for general cases. Jaffe and Cassereau [27] proposed frequencyhopping modulation for a simultaneous transmission system for sonar imaging. However, conventional algorithms, before SEABED, require long calculation time, which negates the fast measurement achieved by multiple transmissions. We achieve simultaneous transmission with multiple antennas by adopting codes with low cross-correlations, in this instance, Gold sequences, which do not require synchronization [28]. This UWB radar system with PN sequences can observe multiple echoes with multiple paths with a single snapshot observation. Using simultaneous transmission, we can take full advantage of the high-speed processing of the SEABED algorithm. 


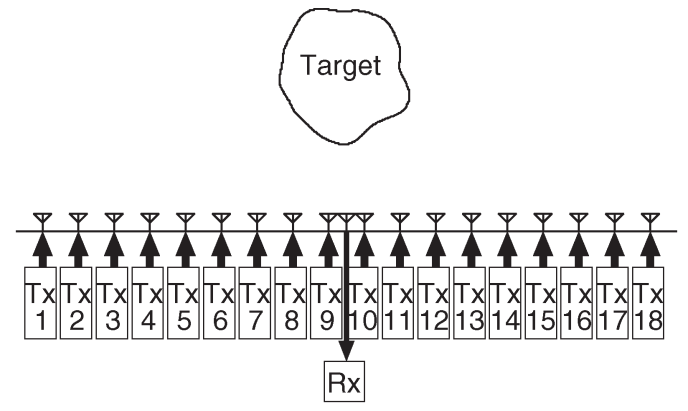

Fig. 1. System model.

We also propose a set of sequences that suppress the range sidelobes of the direct waves without scattering. Direct waves can be measured prior to the actual measurement of targets and can be subtracted from the received data to suppress their effect. This subtraction process works well for systems with high stability, while it cannot completely suppress direct waves for low-cost systems because of their timing jitters. The residual components of the direct waves after the subtraction process cannot be neglected and degrade the imaging quality. Optimizing the set of sequences used to obtain the best quality image is therefore indispensable. The proposed set of sequences improves image quality when compared to those generated with conventional sequences.

\section{SySTEM MOdEL}

An antenna array radar system is assumed as in Fig. 1. Each antenna in the array is assumed to be omnidirectional. It is assumed that $M$ transmitting antennas and one receiving antenna are used. The PN sequences assigned to each transmitting antenna are simultaneously transmitted, and all echoes are received by the receiving antenna. We adopt Gold sequences as transmitted PN codes due to the great number available. The baseband UWB PN signals are transmitted without a carrier. The received signals are A/D converted and stored in memory. For simplicity, a 2-D system with TE mode waves is discussed. The assumed targets have clear boundaries with almost uniform permittivity.

We express the real space in $(x, y)$ in $x-y$ coordinates. Here, $x$ and $y>0$ are normalized by the center wavelength of the transmitting signal $\lambda$. We assume that the receiving antenna is at the origin $(x, y)=(0,0)$ and transmitting antennas are on the $x$-axis. We apply a matched filter of the PN sequence assigned to the transmitting antenna at $(x, y)=(2 X, 0)$ to the received data, and we define $g(X, Y)$ as the output signal of the filter. Here, we define $Y$ with time $t$ and the speed of the radio waves $c$ as $Y=c t /(2 \lambda)$. The received data are expressed as $(X, Y)$, and the target shapes are expressed as $(x, y)$. We define a data space as the space expressed by $(X, Y)$, and the equiphase surface of $g(X, Y)$ is called a quasi-wavefront and is expressed in the data space, which is used to obtain the image in the SEABED algorithm. We normalize $X$ by $\lambda$ and $Y$ by the chip duration of the PN signals. The transform from $(X, Y)$ to $(x, y)$ corresponds to the imaging discussed in this paper.

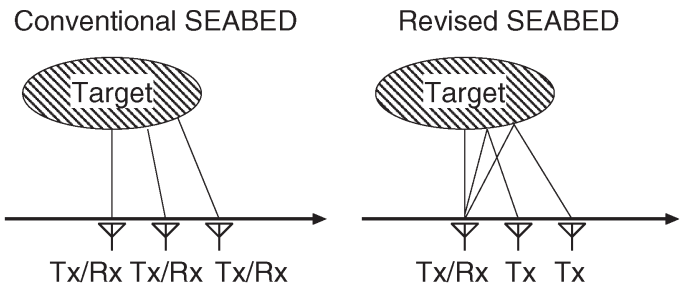

Fig. 2. Antenna layout for conventional and revised SEABED algorithms.

\section{SEABED ALGORITHM FOR RADAR IMAGING}

\section{A. Conventional SEABED Algorithm}

The SEABED algorithm is based on a reversible transform between the target shape and the received data suited to imaging with UWB radar. The SEABED algorithm can directly estimate the target shape with the inverse transform, which is the strict mathematical solution of the inverse scattering problem.

The conventional SEABED algorithm assumes a monostatic radar where the receiving antenna is in the same place as the transmitting antenna $(X, 0)$. The left-hand side of Fig. 2 shows the assumed antenna arrangement for the conventional SEABED algorithm. Under this assumption, the transform from a point $(x, y)$ on the target to the corresponding point on the quasi-wavefront is expressed as [7]

$$
\begin{aligned}
& X=x+y d y / d x \\
& Y=y \sqrt{1+(d y / d x)^{2}}
\end{aligned}
$$

which is called the boundary scattering transform (BST).

The inverse of the BST is expressed as

$$
\begin{aligned}
& x=X-Y d Y / d X \\
& y=Y \sqrt{1-(d Y / d X)^{2}}
\end{aligned}
$$

which is called the inverse BST (IBST). Here, the condition $|d Y / d X| \leq 1$ has to be satisfied because $y$ in (4) should be a real number. We apply this inverse transform to the quasiwavefronts extracted from the received signals and obtain the radar image.

\section{B. Revised SEABED Algorithm}

The radar system dealt with in this paper uses multiple transmitting antennas and a single receiving antenna. The layout of the antennas in this system is different to that in the system described when discussing the SEABED algorithm in the previous section. Therefore, we cannot directly use the IBST from the conventional SEABED algorithm. The righthand side of Fig. 2 shows the assumed antenna arrangement in this paper. The receiving antenna is at $(0,0)$, and the transmitting antenna is at $(2 X, 0)$; these definitions simplify the following equations. Here, we derive a revised IBST for the current antenna arrangement. 
The forward transform, revised BST (RBST) is easily derived as

$$
\begin{aligned}
& X=\frac{\left(x^{2}-y^{2}\right) \dot{y}-x y\left(1-\dot{y}^{2}\right)}{2 x \dot{y}-y\left(1-\dot{y}^{2}\right)} \\
& Y=\frac{1}{2}\left\{\sqrt{x^{2}+y^{2}}+\sqrt{y^{2}+\frac{\left(x^{2}+y^{2}\right)^{2} \dot{y}^{2}}{\left(y-2 x \dot{y}-y \dot{y}^{2}\right)^{2}}}\right\}
\end{aligned}
$$

where $(x, y)$ is a scattering center point on the target surface and $\dot{y}=d y / d x$ is defined.

The ellipse with its major axis $Y$ and that focuses at $(0,0)$ and $(2 X, 0)$ is $F(x, y, X)=0$, where $F(x, y, X)$ is expressed as

$$
F(x, y, X)=\frac{(x-X)^{2}}{Y^{2}}+\frac{y^{2}}{Y^{2}-X^{2}}-1
$$

where $Y$ is not included in the parameters of $F$ because it depends on $X$. The envelope of the group of ellipses for various values of $X$ satisfies the following:

$$
\begin{aligned}
F(x, y, X) & =0 \\
\partial F(x, y, X) / \partial X & =0
\end{aligned}
$$

where the partial derivative by $X$ in the equation means that it is independent of $x$ and $y$, not of $Y$. By solving these equations for $x$ and $y$, we derive the inverse RBST (IRBST) as

$$
\begin{aligned}
& x=\frac{\left(X^{2}+Y^{2}\right) \dot{Y}-2 X Y}{X \dot{Y}-Y} \\
& y=\left|\frac{Y^{2}-X^{2}}{Y-X \dot{Y}}\right| \sqrt{1-\dot{Y}^{2}}
\end{aligned}
$$

where $\dot{Y}=d Y / d X$ is defined. This pair of equations is the inverse transform of the forward transform RBST in (5) and (6). This IRBST is used in the investigations in the following.

The systems assumed in this study and in [25] are both bistatic radar systems, but the models are different. Note that the RBST and IRBST transforms are independent of another pair of transforms, bistatic BST and IBST [25]. The bistatic BST and IBST assume a system with a pair of antennas with a fixed interval, where the pair of antennas scan simultaneously. Conversely, the system discussed in this study uses a fixed receiving antenna with a scanned transmitting antenna, where the antenna interval changes with scanning.

\section{PN SEQUENCES}

Gold sequences are used as the transmitted waveforms in this paper. These sequence are generated from a pair of $\mathrm{M}$-sequences. The M-sequences are generated by a linear feedback shift register (LFSR) [29] with $k$ registers, whose coefficient polynomial is a primitive polynomial in a Galois field [(2)], which has a maximum period of $2^{k}-1$. The Gold sequences are generated by the exclusive summation of a preferred pair of M-sequences. We can make use of an arbitrary relative shift between the pair of $\mathrm{M}$-sequences and obtain $2^{k}+1$ types of Gold sequences including the original preferred pair by applying periodic shifting. The cross-correlation between Gold
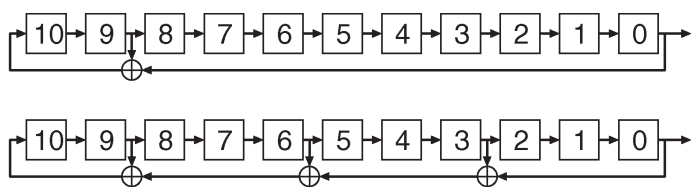

Fig. 3. Assumed LFSR for the preferred pair of M-sequences.

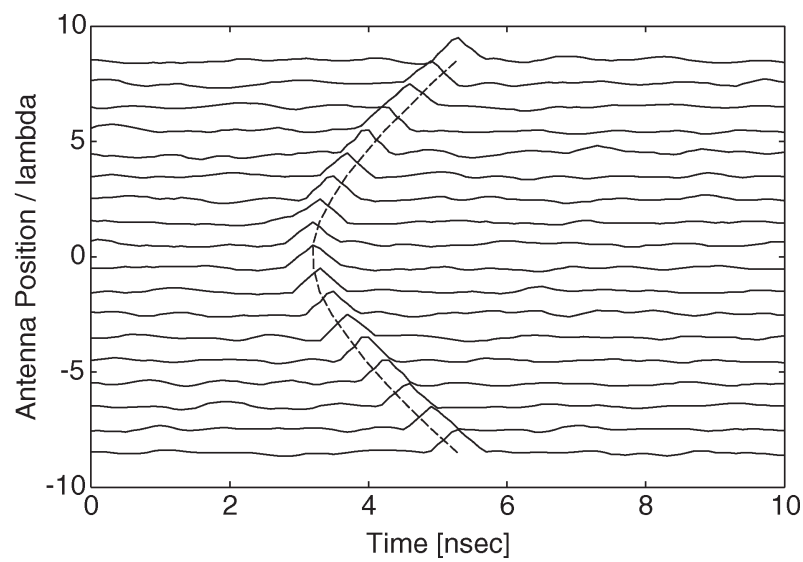

Fig. 4. Received signals for a simultaneous transmission system without direct waves.

sequences is not zero, which causes a certain level of range sidelobes. Therefore, the signal to interference and noise power ratio $S /(I+N)$ has a floor for large $S / N$.

In this paper, we assume that a preferred pair of $\mathrm{M}$-sequences $M_{1}[n]$ and $M_{2}[n](n=1,2, \ldots, 2047)$ are generated by the primitive polynomials

$$
\begin{aligned}
& p_{1}(a)=a^{11}+a^{9}+1 \\
& p_{2}(a)=a^{11}+a^{9}+a^{6}+a^{3}+1 .
\end{aligned}
$$

Here, we set all the initial register values to 1 . The LFSRs for these polynomials are shown in Fig. 3. The $i$ th Gold sequence is generated from these $\mathrm{M}$-sequences as

$$
G_{i}[n]=M_{1}[n]+M_{2}[n+i] \quad(\bmod 2) .
$$

We call $G_{i}[n]$ the $i$ th Gold sequence in the following sections.

\section{Image Degradation by Direct WaVes}

\section{A. Image Obtained Without Direct Waves}

We present an applied example of the proposed radar system and the revised SEABED algorithm. We allocate the $0,1, \ldots, 17$ th Gold sequences to 18 transmitting antennas, using the $G_{0}, G_{1}, \ldots, G_{17}$ as defined in (14) with a time offset of zero. It is assumed that the chiprate is $2.5 \mathrm{Gchip} / \mathrm{s}$, and the antenna interval is $0.12 \mathrm{~m}$, corresponding to one chip duration. The received signals are calculated with a simple ray-tracing method with frequency-independent propagations without noise for simplicity.

The solid line in Fig. 4 shows the output of the matched filter for the sequences assigned to the transmitting antennas. This figure shows the signals with the offset for each antenna position for clarity. The extracted quasi-wavefront is shown as a broken line. The data received by this system are almost the 


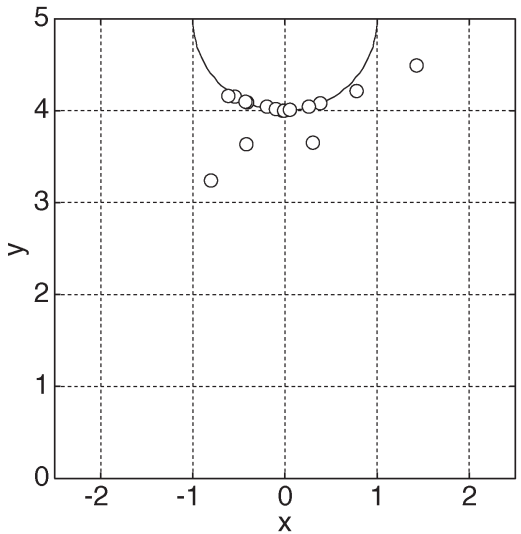

Fig. 5. Estimated image for a simultaneous transmission system without direct waves.

same as the data from scanning a transmitting antenna with a fixed receiving antenna except for the interference component. Here, the random component in the background is caused by the range sidelobes of the auto- and cross-correlations of the Gold sequences as noise is neglected in this numerical simulation. Fig. 5 shows the image estimated by the revised SEABED algorithm with received signals in Fig. 4. As aforementioned, $x$ and $y$ are normalized by $\lambda$. The image points estimate the target shape, assumed to be a circular metallic object, with good accuracy. In this figure, a few points have large errors; this is caused by the range sidelobe described previously.

\section{B. Image Obtained With Direct Waves}

In the previous section, we investigated the imaging performance of a code-division UWB radar system with scattering waves only, without evaluating performance with direct waves. Direct waves are the waves received directly from the transmitting antenna without scattering. The effect of direct waves is moderate if high-gain antennas, like horn antennas, are used. However, small antennas are required to obtain the entire image from a large area, meaning we cannot neglect the influence of direct waves on imaging performance. In this section, we quantitatively investigate the influence of direct waves on the imaging performance of the proposed radar system.

Direct waves are measured prior to the actual measurement of the target and are subtracted from the measured data. Following this process, the residual components of the direct waves are contained in the signal and are referred to as "direct waves" in the following explanation. Fig. 6 shows the direct waves received after the despreading process. We see that range sidelobes are uniformly distributed around the peaks. Fig. 7 shows the received signals with both direct and scattering waves, with the power of the direct waves set to about $10 \mathrm{~dB}$ larger than that of the echoes. A part of the echoes' waveforms is degraded by the sidelobe of the direct waves. Fig. 8 shows the estimated target shape from the revised SEABED algorithm for these signals. We see that the estimation error is larger than that for the image in Fig. 5, which means that we cannot ignore the effect of direct waves. The inverse transform IRBST in (10) and (11) contains derivative operations, which make the image sensitive to random components. This is a critical problem in

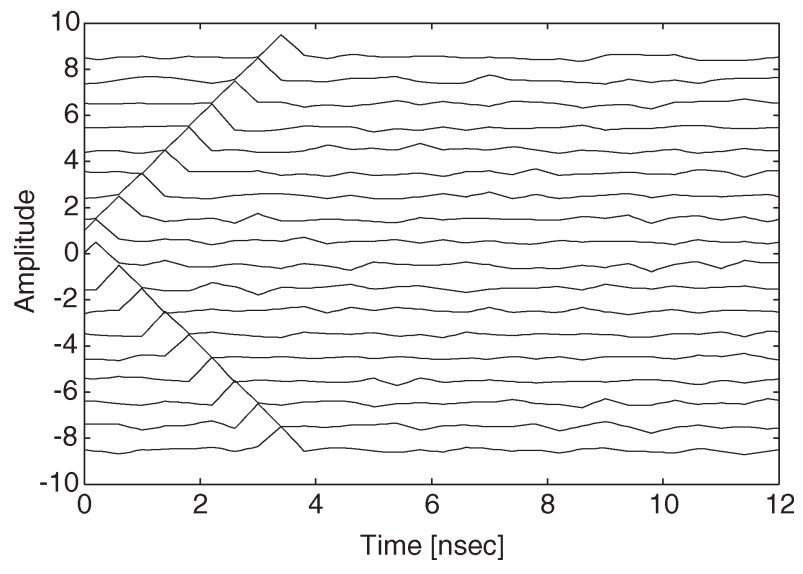

Fig. 6. Direct waves received by a simultaneous transmission system.

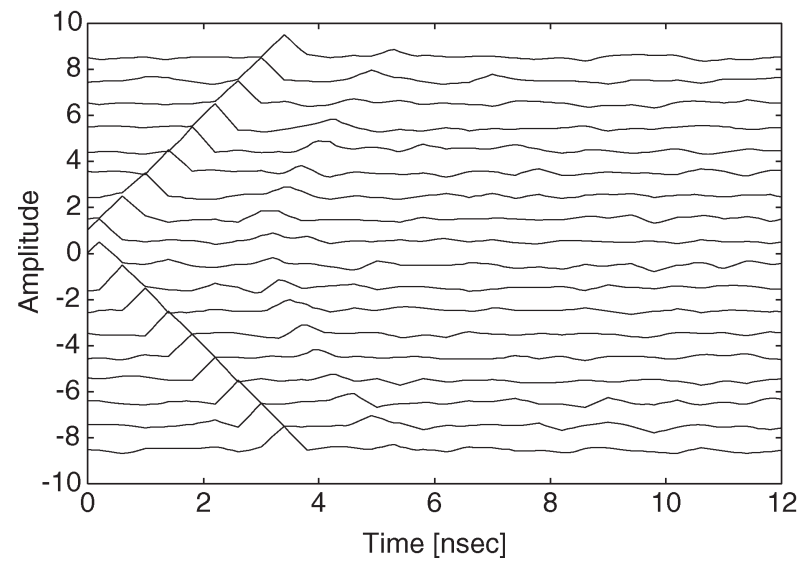

Fig. 7. Received signals with direct waves.

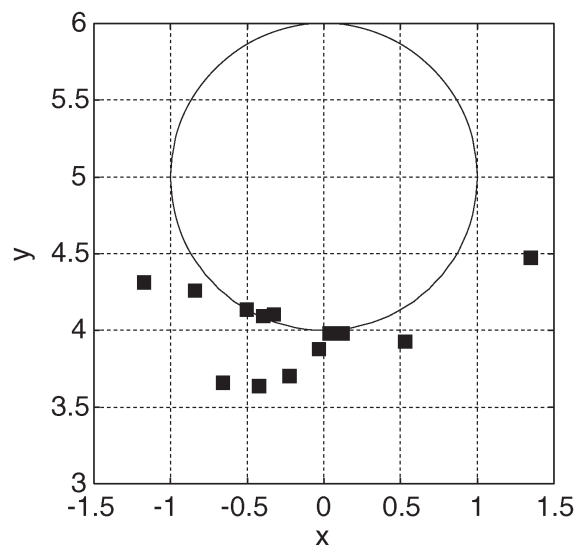

Fig. 8. Estimated image from conventional code using received signals with direct waves.

the application of PN codes to simultaneous transmission array systems for near target imaging.

\section{Optimum Spreading Sequences FOR UWB RADAR}

\section{A. Evaluation Function to Suppress Range Sidelobes}

To resolve the image degradation caused by direct waves, a suitable set of sequences was sought to suppress the sidelobes of the direct waves. The receiving timing of each sequence 


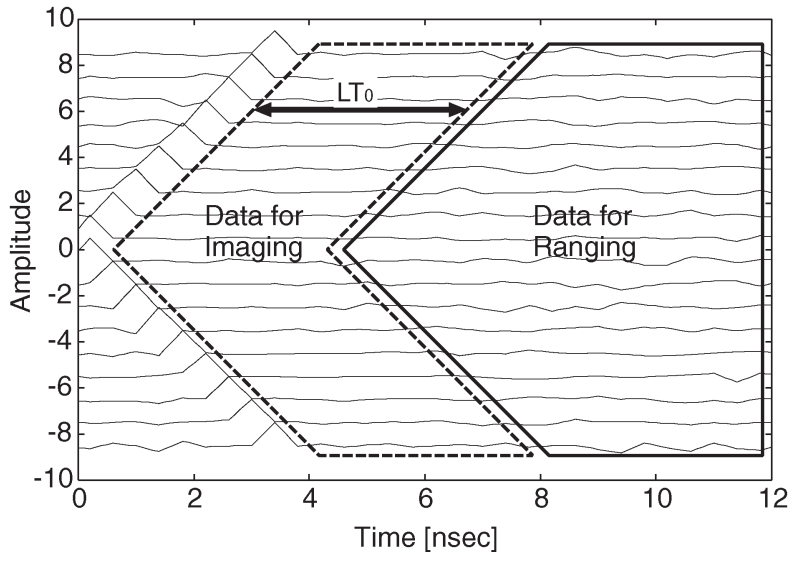

Fig. 9. Data areas used for imaging and ranging.

is fixed because the distance between the transmitting and receiving antenna is fixed. Making use of this characteristic, we searched for a set of sequences whose direct waves' range sidelobes cancel each other out. This process is similar to the pair of complimentary sequences that cancel the sidelobes of autocorrelation functions. We selected 18 suitable candidates from 2049 types of Gold sequences. Additionally, we selected a suitable time shift for each sequence as the transmitting timing can be arbitrarily set. We suppress the sidelobes in the area near the antennas only because it is not necessary to suppress all sidelobes. The SEABED algorithm does not work for distant targets because the locations of the scattering centers become almost independent of the position of the antennas at distance. We only estimate the distance to the target for shapes in the far-field and distant sidelobes do not need to be suppressed. However, we do estimate the target shape for targets in the near field. We can average multiple signals to suppress the range sidelobes, which enhances the ranging accuracy for targets in the far field. Fig. 9 shows the data areas used for imaging and ranging as described previously. Gold sequences were selected to guarantee impulse-shaped autocorrelation functions and lowlevel asynchronous cross-correlation functions.

The code $\boldsymbol{c}_{m}$ with length $N$ is assigned to the $m$ th transmitting antenna and is expressed as

$$
\boldsymbol{c}_{m}=\left[c_{m, 1}, c_{m, 2}, \ldots, c_{m, N}\right] .
$$

The received signal $s_{m}^{\mathrm{T}}$ from the $m$ th transmitting antenna is expressed as

$$
s_{m}^{\mathrm{T}}(t)=\sum_{i=1}^{N} c_{m, i} p\left(t-i T_{0}\right)
$$

where $T_{0}$ is the chip duration and $p(t)$ is the impulse response of the total system which is assumed to satisfy $p(t)=0$ for $|t|>T_{0} / 2$. The received signal $s(t)$ is

$$
s(t)=\sum_{m=1}^{M} s_{m}^{\mathrm{T}}(t)=\sum_{m=1}^{M} \sum_{i=1}^{N} c_{m, i} p\left(t-i T_{0}\right)
$$

where $M$ is the number of sequences (or transmitting antennas) and the delay time for each code is assumed to be zero for simplicity. Here, complete synchronization is assumed. To obtain the echo $s_{m}(t)$ for the $m$ th transmitting code, we calculate the cross-correlation function between $s(t)$ and the transmitted signal $s_{m}^{\mathrm{T}}\left(t-d_{m}\right)$ for the $m$ th code as

$$
\begin{aligned}
s_{m}(t)= & \int s(\tau) s_{m}^{\mathrm{T}}\left(\tau-t+d_{m}\right) d \tau \\
= & \sum_{n=1}^{M} \sum_{i=1}^{N} \sum_{j=1}^{N} c_{n, i} c_{m, j} \\
& \cdot \int p\left(\tau-i T_{0}\right) p\left(\tau-t-j T_{0}+d_{m}\right) d \tau \\
= & \sum_{n=1}^{M} \sum_{i=1}^{N} \sum_{j=1}^{N} c_{n, i} c_{m, j} R_{p}\left(t+(j-i) T_{0}-d_{m}\right)
\end{aligned}
$$

where $d_{m}$ is the time delay from the $m$ th transmitting and the receiving antennas, and $R_{p}(t)$ is the autocorrelation function of $p(t)$ and satisfies $R_{p}(t)=0$ for $|t|>T_{0}$. The following is derived by assuming the periodical extension of the definition of $c_{m, i}$ as $c_{m, i+N}=c_{m, i}$ for any $m$ and $i$ :

$$
\begin{aligned}
s_{m}(t) & =\sum_{n=1}^{M} \sum_{l=1}^{N} \sum_{i=1}^{N} c_{n, i} c_{m, i+l} R_{p}\left(t-l T_{0}-d_{m}\right) \\
& =\sum_{l=0}^{N} R_{p}\left(t-l T_{0}-d_{m}\right) \sum_{n=1}^{M} r_{m, n}(l)
\end{aligned}
$$

where $r_{m, n}(l)$ is the cross-correlation function of sequences $\boldsymbol{c}_{m}$ and $\boldsymbol{c}_{n}$, derived by changing variables as $l=i-j$.

The range sidelobe level for $T_{0} \leq t \leq L T_{0}$ is expressed as

$$
\begin{aligned}
& \int_{T_{0}+d_{m}}^{L T_{0}+d_{m}}\left|s_{m}(t)\right|^{2} d t \\
& \quad=\int_{T_{0}}^{L T_{0}}\left|\sum_{l=0}^{N} R_{p}\left(t-l T_{0}\right) \sum_{n=1}^{M} r_{m, n}(l)\right|^{2} d t \\
& \simeq R_{0}^{2} \sum_{l=1}^{N}\left\{\sum_{n=1}^{M} r_{m, n}(l)\right\}^{2}
\end{aligned}
$$

where $R_{0}$ is a constant determined by the amplitude of $R_{p}(t)$. The approximation in (24) uses the condition $R_{p}\left(t-l T_{0}\right)=0$ for $T_{0}<\left|t-l T_{0}\right|$. In this paper, we aim to find the set of Gold sequences that optimally minimizes the sum of the range sidelobe levels for $m=1,2, \ldots, M$.

We set the evaluation function to select the sequences as

$$
\operatorname{minimize}_{\boldsymbol{c}_{1}, \boldsymbol{c}_{2}, \ldots, \boldsymbol{c}_{M}} \sum_{m=1}^{M} \sum_{l=1}^{L}\left\{\sum_{n=1}^{M} r_{m, n}(l)\right\}^{2}
$$

where $L$ is the number of chips where the sidelobes are suppressed. We set $M=18$ and $L=9$ here.

As an example, setting the chip rate to $2.5 \mathrm{Gchip} / \mathrm{s}$ suppresses the range sidelobes within $1 \mathrm{~m}$ of the antennas. To check for all the possible combinations of (25), we would have to calculate the evaluation function $T_{\text {cal }}=2049 C_{18} \cdot 2047^{18}$ times. 


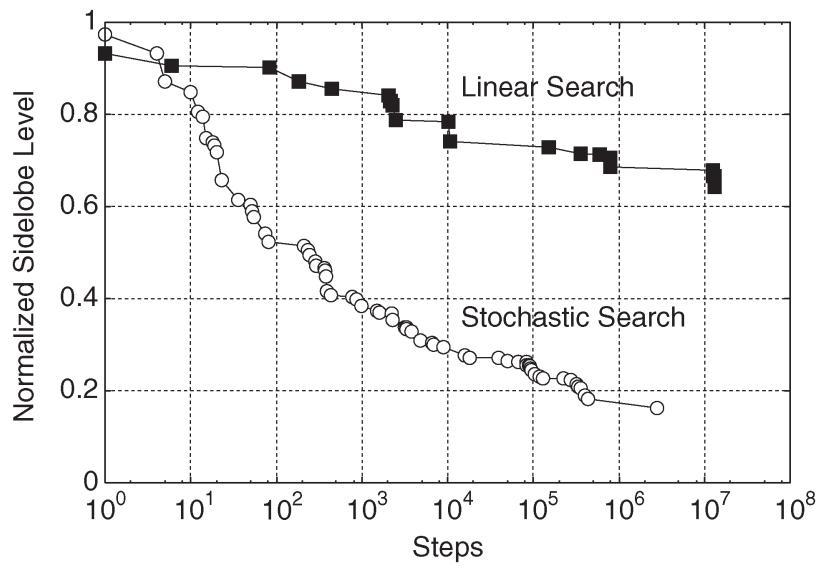

Fig. 10. Normalized sidelobe level versus calculation steps.

We estimate that this calculation needs about $10^{93}$ years with a single Xeon 2.8-GHz processor, which is clearly not realistic. Therefore, our objective must be to find a suboptimum solution for this problem.

\section{B. Suboptimal Sequences to Suppress Direct Waves}

We adopt a stochastic search algorithm for the optimization, in which random instances of the cost function (25) are generated and the smallest is adopted. The optimization procedure with stochastic search algorithm is described in the following, where $\operatorname{ran}(n)$ is a uniform random integer between 1 and $n$.

1) Generate $\operatorname{ran}\left(2^{k}+1\right) M$ times. Go to 2$)$.

2) If there is a duplicate pair of numbers in the $M$ numbers, go to 1). Otherwise, set the $M$ numbers as the initial code numbers for the antennas and go to 3 ).

3) Generate $\operatorname{ran}\left(2^{k}-1\right) M$ times. Set the numbers as the initial cyclic shifts for the antennas and go to 4$)$.

4) Calculate and store the evaluation value as the minimum value and store the set of sequences. Go to 5$)$.

5) Generate $\operatorname{ran}(M), \operatorname{ran}\left(2^{k}+1\right)$, and $\operatorname{ran}\left(2^{k}-1\right)$. Set the code number and shift for the $\operatorname{ran}(M)$ th antenna to $\operatorname{ran}\left(2^{k}+1\right)$ and $\operatorname{ran}\left(2^{k}-1\right)$, respectively. Go to 6$)$.

6) If there is a duplicate pair of numbers in the antenna number, go to 5). Otherwise, calculate the evaluation value and go to 7).

7) If the calculated evaluation value is smaller than the stored minimum value, substitute the new value for the minimum value and store the sequences. Otherwise, cancel the previous change by substituting the stored sequences for the present sequences. Go to 5).

Here, $2^{k}-1$ is the period of the Gold sequences, and $k=11$ and $M=18$ are assumed in the following numerical simulation. After a set number of iterations, the final optimum sequences from the aforementioned procedure can be adopted for the proposed radar system. The number of iterations is empirically set to an optimal $10^{8}$. The calculation time for the search depends approximately on the number of calculations of the evaluation function. Fig. 10 shows the relationship between the evaluation value and the number of calculations. We show the results of both the linear search and the stochastic search
TABLE I

OPTIMIZED SET OF GOLD SEQUENCES FOR A UWB RADAR SYSTEM With LINEAR ANTENNA ARRAY

\begin{tabular}{|c||c|c|}
\hline Antenna & Code number & Time shift \\
\hline \hline 1 & 655 & 1370 \\
\hline 2 & 1739 & 963 \\
\hline 3 & 1584 & 90 \\
\hline 4 & 1474 & 384 \\
\hline 5 & 1367 & 1478 \\
\hline 6 & 677 & 7 \\
\hline 7 & 614 & 780 \\
\hline 8 & 194 & 587 \\
\hline 9 & 1357 & 1372 \\
\hline 10 & 532 & 1619 \\
\hline 11 & 779 & 1027 \\
\hline 12 & 1958 & 1868 \\
\hline 13 & 1403 & 2007 \\
\hline 14 & 528 & 2035 \\
\hline 15 & 1803 & 1441 \\
\hline 16 & 1348 & 983 \\
\hline 17 & 1120 & 1125 \\
\hline 18 & 264 & 385 \\
\hline
\end{tabular}

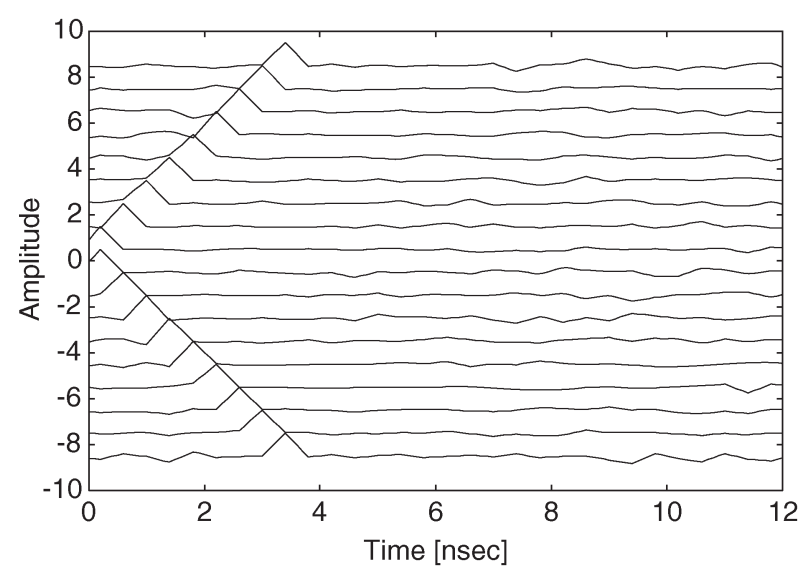

Fig. 11. Direct waves with proposed sequences.

algorithm in this figure. The result shows that the stochastic search algorithm effectively solves our optimization problem. The stochastic search algorithm suppresses the normalized sidelobe level to about $16 \%$ compared to arbitrarily chosen sequences. Adopting the optimized sequences, we can improve the $\mathrm{S} / \mathrm{I}$ (signal-to-interference ratio) by $8 \mathrm{~dB}$ without additional costs for the system. Table I shows the optimized set of Gold sequences with the code number and the time shift. The code number corresponds to (14). Additional time shift is needed depending on the propagation delay between each transmitting antenna and the receiving antenna for actual application to synchronize all codes at the position of the receiving antenna.

Note that the optimization method used with the stochastic search is not guaranteed to be the best solution for this kind of problem. Other methods including an algorithm for approximating the solution to a combinatorial optimization problem should be employed and investigated in future studies.

\section{Imaging With the Proposed Sequences}

Let us investigate the imaging performance of the proposed radar system and sequences. Fig. 11 shows the output signals 


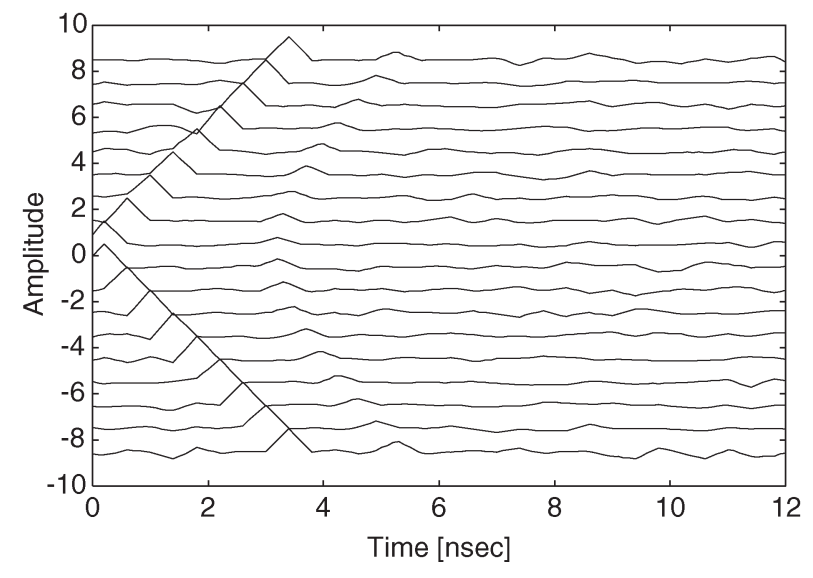

Fig. 12. Received signals with direct waves.

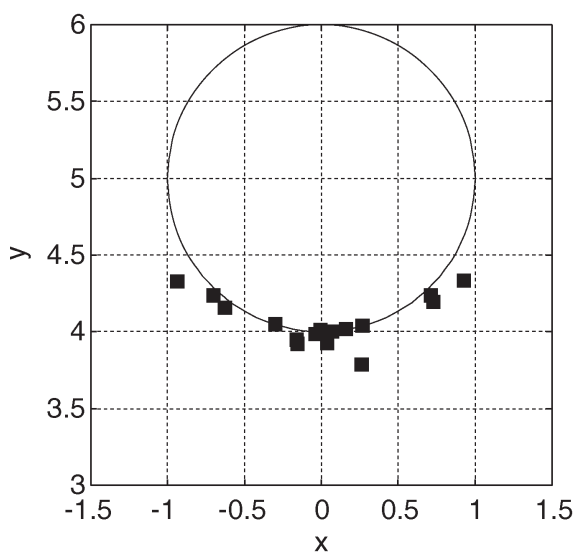

Fig. 13. Estimated image with the proposed codes using received signals with direct waves.

TABLE II

ESTIMATION RMS ERRORS OF PROPOSED AND CONVENTIONAL CODES

\begin{tabular}{|c||c|c|}
\hline & Proposed & Conventional \\
\hline \hline Without direct-wave & $6.55 \mathrm{psec}$ & $9.18 \mathrm{psec}$ \\
\hline With direct-wave & $9.91 \mathrm{psec}$ & $65.5 \mathrm{psec}$ \\
\hline
\end{tabular}

after applying the matched filter to the direct waves with the proposed sequences. The sidelobe levels become low within the area closer than $3.6 \mathrm{~ns}$ to the peaks when compared to Fig. 6 . Fig. 12 shows the echoes and the direct waves with the proposed sequences. We clearly see the peaks of the echoes compared to Fig. 7, which demonstrates the improvement in S/I. Fig. 13 shows the image estimated with the SEABED algorithm revised with the selected sequences. The accuracy of the estimation is improved compared to the image obtained with conventional sequences in Fig. 8.

Table II shows the root-mean-square (rms) error of the estimation of the quasi-wavefront, comparing the proposed and conventional code sets. This table shows that the error is strongly influenced by direct waves for conventional codes, while the error is acceptable for the proposed codes. The transform IRBST contains derivative operations, which are sensitive to random components, and the transform has a nonlinear property. The relatively small estimation errors of the quasi-wavefront are amplified by these factors, which, in turn, produce the large degradation in the estimated images. This kind of degradation can be reduced by stabilization techniques as in [13] and [15].

\section{CONCLUSION}

In this paper, we introduce PN signals with Gold sequences as replacements for conventional short pulses in UWB radar imaging. This enables us to simultaneously transmit signals with multiple antennas. This radar system can gather observation data quickly, maintaining the speed advantages of the SEABED algorithm. The SEABED algorithm has been revised to deal with a fixed receiving antenna and multiple-arrayed transmit antennas. Although this proposed radar system suffers from range sidelobes due to auto- and cross-correlations between PN codes, the quality of the estimated target image is sufficient for most applications. The efficacy of the proposed radar system and algorithm was demonstrated with numerical simulations.

As future work, we are planning the experimental verification of the proposed radar system by introducing an Anritsu MP1800 digital signal generator, an Agilent Technology 86100C wideband receiver, and an 18-element UWB antenna array.

\section{REFERENCES}

[1] E. J. Bond, X. Li, S. C. Hagness, and B. D. van Veen, "Microwave imaging via space-time beamforming for early detection of breast cancer," IEEE Trans. Antennas Propag., vol. 51, no. 8, pp. 1690-1705, Aug. 2003.

[2] R. M. Narayanan, X. Xu, and J. A. Henning, "Radar penetration imaging using ultra-wideband (UWB) random noise waveforms," Proc. Inst. Elect. Eng.-Radar Sonar Navig., vol. 151, no. 3, pp. 143-148, Jun. 2004.

[3] J. van der Kruk, C. P. A. Wapenaar, J. T. Fokkema, and P. M. van den Berg, "Three-dimensional imaging of multicomponent ground-penetrating radar data," Geophysics, vol. 68, no. 4, pp. 1241-1254, Jul./Aug. 2003.

[4] C. J. Leuschen and R. G. Plumb, "A matched-filter-based reverse-time migration algorithm for ground-penetrating radar data," IEEE Trans. Geosci. Remote Sens., vol. 39, no. 5, pp. 929-936, May 2001.

[5] A. Franchois and C. Pichot, "Microwave imaging-complex permittivity reconstruction with a Levenberg-Marquardt method," IEEE Trans. Antennas Propag., vol. 45, no. 2, pp. 203-215, Feb. 1997.

[6] T. J. Cui, Y. Qin, G. L. Wang, and W. C. Chew, "High-order inversion formulas for low-frequency imaging of 2D buried targets," in Proc. IEEE Antennas Propag. Soc. Int. Symp., 2004, vol. 1, pp. 189-192.

[7] T. Sakamoto and T. Sato, "A target shape estimation algorithm for pulse radar systems based on boundary scattering transform," IEICE Trans. Commun., vol. E87-B, no. 5, pp. 1357-1365, May 2004.

[8] T. Sakamoto and T. Sato, "Fast imaging of a target in inhomogeneous media for pulse radar systems," in Proc. IEEE Int. Geosci. Remote Sens. Symp., Sep. 2004, vol. 3, pp. 2070-2073.

[9] T. Sakamoto and T. Sato, "A phase compensation algorithm for highresolution pulse radar systems," IEICE Trans. Commun., vol. E87-B, no. 11, pp. 3314-3321, Nov. 2004.

[10] T. Sakamoto and T. Sato, "A phase compensation algorithm for highresolution pulse radar systems," in Proc. Int. Symp. Antennas Propag., Aug. 2004, pp. 585-588.

[11] T. Sakamoto and T. Sato, "A fast algorithm of 3-dimensional imaging for pulse radar systems," in Proc. IEEE AP-S Int. Symp. USNC/URSI Nat. Radio Sci. Meeting, Jun. 2004, vol. 2, pp. 2099-2102.

[12] S. Kidera, T. Sakamoto, and T. Sato, "A high-resolution imaging algorithm based on scattered waveform estimation for UWB pulse radar systems," in Proc. IEEE Int. Geosci. Remote Sens. Symp., Jul. 2005, pp. $1725-1728$.

[13] T. Sakamoto, S. Kidera, T. Sato, T. Mitani, and S. Sugino, "An experimental study on a fast imaging algorithm for UWB pulse radar systems," in Proc. IEEE AP-S Int. Symp. USNC/URSI Nat. Radio Sci. Meeting, Jul. 2005, pp. 690-693. 
[14] T. Sakamoto, S. Kidera, T. Sato, T. Mitani, and S. Sugino, "An experimental study on a fast and accurate 3-D imaging algorithm for UWB pulse radar systems," in Proc. 28th Gen. Assembly Int. URSI, Oct. 2005. F05.7.

[15] T. Sakamoto, "A 2-D image stabilization algorithm for UWB pulse radars with fractional boundary scattering transform," IEICE Trans. Commun., vol. E90-B, no. 1, pp. 131-139, Jan. 2007.

[16] T. Sakamoto, "A fast algorithm for 3-dimensional imaging with UWB pulse radar systems," IEICE Trans. Commun., vol. E90-B, no. 3, pp. 636644, Mar. 2007.

[17] S. Hantscher, A. Reisenzahn, and C. G. Diskus, "Through-wall imaging with a 3-D UWB SAR algorithm," IEEE Signal Process. Lett., vol. 15, no. 1, pp. 269-272, 2008.

[18] D. W. Winters, J. D. Shea, E. L. Madsen, G. R. Frank, B. D. Van Veen, and S. C. Hagness, "Estimating the breast surface using UWB microwave monostatic backscatter measurements," IEEE Trans. Biomed. Eng., vol. 55, no. 1, pp. 247-256, Jan. 2008.

[19] M. Helbig, M. Hein, U. Schwarz, and J. Sachs, "Preliminary investigations of chest surface identification algorithms for breast cancer detection," in Proc. Int. Conf. Ultra- Wideband, Sep. 2008, pp. 195-198.

[20] T. Counts, A. C. Gurbuz, W. R. Scott, Jr., J. H. McClellan, and K. Kim, "Multistatic ground-penetrating radar experiments," IEEE Trans. Geosci. Remote Sens., vol. 45, no. 8, pp. 2544-2553, Aug. 2007.

[21] M. Dehmollaian and K. Sarabandi, "Refocusing through building walls using synthetic aperture radar," IEEE Trans. Geosci. Remote Sens., vol. 46, no. 6, pp. 1589-1599, Jun. 2008.

[22] A. G. Yarovoy, T. G. Savelyev, P. J. Aubry, P. E. Lys, and L. P. Ligthart, "UWB array-based sensor for near-field imaging," IEEE Trans. Microw. Theory Tech., vol. 55, no. 6, pp. 1288-1295, Jun. 2007.

[23] S. Masuyama and A. Hirose, "Walled LTSA array for rapid, high spatial resolution, and phase-sensitive imaging to visualize plastic landmines," IEEE Trans. Geosci. Remote Sens., vol. 45, no. 8, pp. 2536-2543, Aug. 2007.

[24] J. L. Schwartz and B. D. Steinberg, "Ultrasparse, ultrawideband arrays," IEEE Trans. Ultrason., Ferroelectr., Freq. Control, vol. 45, no. 2, pp. 376393, Mar. 1998.

[25] S. Kidera, Y. Kani, T. Sakamoto, and T. Sato, "A fast and highresolution 3-D imaging algorithm with linear array antennas for UWB pulse radars," IEICE Trans. Commun., vol. E91-B, no. 8, pp. 2683-2691, Aug. 2008.

[26] S. Ishigami, H. Yanagida, Y. Tamura, C. Ishihara, and N. Okada, “Observation of dynamic structure using ultrasound 3D imaging system with encoded wave front," Jpn. J. Appl. Phys., vol. 42, no. 5B, pp. 3276-3280, May 2003.

[27] J. S. Jaffe and P. M. Cassereau, "Multibeam imaging using spatially variant insonification," J. Acoust. Soc. Amer, vol. 83, no. 4, pp. 14581464, Apr. 1988
[28] T. Sakamoto and T. Sato, "Code-division multiple transmission for highspeed UWB radar imaging with array antennas," in Proc. IEEE Antennas Propag. Int. Symp., 2007, pp. 429-432.

[29] D. V. Sarwate and M. B. Pursley, "Crosscorrelation properties of pseudorandom and related sequences," Proc. IEEE, vol. 68, no. 5, pp. 593-619, May 1980.

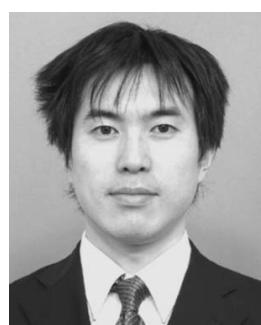

Takuya Sakamoto (S'04-M'05) was born in Nara, Japan, in 1977. He received the B.E. degree from Kyoto University, Kyoto, Japan, in 2000 and the M.I. and Ph.D. degrees from the Graduate School of Informatics, Kyoto University, in 2002 and 2005, respectively.

He is an Assistant Professor with the Department of Communications and Computer Engineering, Graduate School of Informatics, Kyoto University. His current research interest is in ultrawideband radar signal processing.

Dr. Sakamoto is a member of the Institute of Electronics, Information, and Communication Engineers and the Institute of Electrical Engineers of Japan.

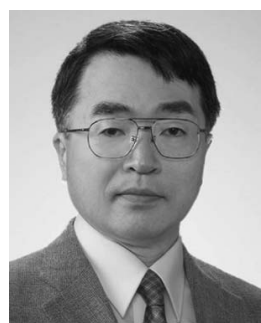

Toru Sato (M'92) received the B.E., M.E., and $\mathrm{Ph} . \mathrm{D}$. degrees in electrical engineering from Kyoto University, Kyoto, Japan, in 1976, 1978, and 1982, respectively.

He has been with Kyoto University since 1983 and is currently a Professor with the Department of Communications and Computer Engineering, Graduate School of Informatics. His major research interests have been the system design and signal processing aspects of atmospheric radars, radar remote sensing of the atmosphere, observations of precipitation using radar and satellite signals, radar observation of space debris, and signal processing for subsurface radar signals.

Dr. Sato is a member of the Institute of Electronics, Information, and Communication Engineers of Japan, the Society of Geomagnetism and Earth, Planetary and Space Sciences, the Japan Society for Aeronautical and Space Sciences, and the American Meteorological Society. He was the recipient of the Tanakadate Prize in 1986. 